\section{The Best Test in the Least Time}

To the Editor:- Hatala et al. made excellent points regarding reliability and accuracy of clinical examinations in their recent article. ${ }^{1}$ However, current practices provide physicians short appointment intervals for patient encounters. The clinician must use the best test with the least time expenditure. As such, for the scenario they presented, I would opt either to administer the Edinburgh Claudication Questionnaire, which has the best positive likelihood ratio $(\mathrm{LR}+=90)$ and next-best negative likelihood ratio (LR $-=0.1$ ), or auscultate the femoral arteries and palpate the posterior tibial arteries (Table 1). The time required for focusing on contour and amplitude would prevent me from palpating the femoral arteries. Similarly, I would not perform the ankle-brachialindex maneuver as it would require that I leave the examination room to locate the Doppler equipment and that I perform additional blood pressure measurements in three of four extremities. I would be comfortable advising Mr. Jones that he was at least twice as likely to have vascular disease based on the presence of femoral bruits or the absence of posterior tibial pulses.

Future studies might include an assessment of time needed to perform the bedside tests listed in Table 1 and an assessment of the value of combining auscultation for femoral bruits and palpation for posterior tibial arteries. Assuming logistic regression using

Table 1. Effect of Likelihood Ratio on Posttest Probability for Peripheral Vascular Disease Using Different Clinical Examination Maneuvers

\begin{tabular}{lccc}
\hline \hline Clinical Finding & $\begin{array}{c}\text { Pretest } \\
\text { Probability }\end{array}$ & $\begin{array}{c}\text { Likelihood } \\
\text { Ratio Positive } \\
\left(\mathrm{LR}^{+}\right)\end{array}$ & $\begin{array}{c}\text { Posttest } \\
\text { Probability }\end{array}$ \\
\hline $\begin{array}{c}\text { Edinburgh } \\
\text { Claudication }\end{array}$ & & & \\
$\quad$ Questionnaire & 0.26 & 90 & 0.97 \\
$\begin{array}{c}\text { Abnormal femoral } \\
\text { pulse }\end{array}$ & 0.26 & 7.1 & 0.71 \\
$\begin{array}{c}\text { Femoral bruit } \\
\text { Abnormal posterior } \\
\text { tibial pulse }\end{array}$ & 0.26 & 4.7 & 0.62 \\
$\begin{array}{c}\text { Abnormal ankle- } \\
\text { brachial index }\end{array}$ & 0.26 & 4.6 & 0.62 \\
\hline
\end{tabular}

JGIM welcomes your letters and comments. Letters may contain brief commentaries on articles published in the Journal, illustrative case reports, general suggestions for improving the Journal, or other information of interest to readers. Letters to the Editor should not exceed 450 words in length and should be sent in triplicate (typed, double-spaced). We cannot guarantee publication and we may edit to improve readability.

You may send your letters to us by mail, fax, or e-mail: JGIM, Veterans Affairs Medical Center (JGIM-111), University and Woodland Avenues, Philadelphia, PA 19104; fax: 215-8234450; e-mail: walklett@mail.med.upenn.edu. backward elimination indicates these tests are independent predictors, ${ }^{2}$ the combination would have a higher LR + or lower LR than either test alone. Perhaps the likelihood ratios would be superior to the Edinburgh Claudication Questionnaire or the anklebrachial-index test.-DAvid A. NARDone, MD, Portland, Ore.

\section{REFERENCES}

1. Hatala R, Smieja M, Kane SL, Cook DJ, Meade MO, Nishikawa J. An evidence-based approach to the clinical examination. J Gen Intern Med. 1997;12:182-7.

2. Holleman DR, Simel DL. Quantitative assessments from the clinical examination: how should clinicians integrate the numerous results? J Gen Intern Med. 1997;12:165-71.

In reply:-The objective of our review was to provide evidence about the strengths and limitations of various aspects of the history and physical examination in the detection of peripheral vascular disease. As Dr. Nardone suggests, when faced with limited examination time and numerous examination maneuvers, it is logical to perform only the individual maneuvers that have the highest accuracy and are not overly time-consuming. It may be helpful to involve allied health professionals in completing maneuvers that are time-consuming, either before or after the physician encounter with the patient.

In examining for peripheral vascular disease, it is plausible that various findings (abnormal femoral or tibial pulses or femoral bruits) are not independent of each other but coexist in individual patients, thus reducing their combined accuracy. Table 2 in our article reveals that although many of the maneuvers are helpful in establishing the presence of peripheral vascular disease because they have high positive likelihood ratios, most do not effectively rule out the presence of significant disease because of inadequate negative likelihood ratios. As Dr. Nardone has highlighted, further studies assessing the accuracy of multiple physical examination maneuvers when completed singly or in combination, should be the next step in the evolution of clinical skills assessments.-Rose Hatala, MD, Hamilton, Ont., Canada.

\section{Quantitative Assessments from the Clinical Examination}

To the Editor:-In the March 1997 issue, Holleman et al. ${ }^{1}$ tested four strategies for using multiple clinical examination items to estimate disease probabilities: (1) traditional combination of multiple likelihood ratios (LRs), (2) single best LR, (3) logistic regression-selected traditional LRs and (4) logistic regression itself. They showed that strategies 3 and 4 were best. The authors could not state whether strategy 4 was better than strategy 3. After reading their well-written article, I had lingering doubts about the premise of the study. 
Strategy 4 (multivariate logistic regression) tests for conditional independence among the predictor variables for predicting the outcome variable. When conditional independence is violated, the regression coefficients are adjusted accordingly. By definition, therefore, the predictive ability of strategy 4 will always be at least as great as strategy 3 , and most often greater. A clinical data set is not needed to show this.

Logistic regression was used in strategy 3 to determine which variables were independently related to the outcome variable. From the ratio of the LRs listed in Table 3, I calculated that auscultated wheezing had the largest of the three regression coefficients. It was also the single predictor variable with the highest LR (and was therefore used in strategy 2). However, logistic regression also identified two other variables that were independently predictive of the outcome. Therefore, if the information from these two variables were added, even using the traditional LR calculation of strategy 2 , it seems obvious that the predictive power could only increase. Is it plausible that strategy 2 could ever be better than strategy 3 ?

Strategy 1 uses the traditional LR method for all seven predictor variables found to be significant in univariate analyses. However, logistic regression (strategies 3 and 4) identified that only three variables were independent predictors. It seems obvious that adding variables without independent predictive ability would only worsen the overall accuracy of prediction. Is it possible, given that only three variables were independent predictors, that strategy 1 could ever be better than any of the others? The logistic regression confirmed the clinical suspicion that patients with one important clinical finding thought to be related to the presence of disease are very likely to have other potentially important clinical findings. It seems unlikely then that clinicians would find strategy 1 plausible for clinical use. Indeed, the predictive ability of clinicians' subjective assessments was better than strategy 1 .

In summary, the article seems to be saying that adjusted combination of independent predictor variables (logistic regression, strategy 4) is better than indiscriminate combination of data (strategy 1), selected univariate analysis (strategy 2), and unadjusted combination of independent predictors (strategy 3). Logistic regression thus does what it is supposed to do. Was there residual doubt regarding this?

Finally, a recent study reported the use of classification and regression tree analysis (CART) to distinguish among emergency department patients with chest pain who might need intensive care. ${ }^{2}$ CART has advantages over logistic regression in that it accounts for interactions between variables. Did the authors consider testing this fifth strategy?-DANIEL J. CHER, MD, Palo Alto VA Health Care System, Palo Alto, Calif.

\section{REFERENCES}

1. Holleman DR, Simel DL. Quantitative assessments from the clinical examination. J Gen Intern Med. 1997; 12:165-71.

2. Goldman L, Cook EF, Johnson PA, Brand DA, Rouan GW, Lee TH. Prediction of the need for intensive care in patients who come to the emergency departments with acute chest pain. N Engl J Med. 1996;334(23): 1498-504.

In reply:-We thank Dr. Cher for his insightful comments about our article. Perhaps he has stated the premises of the paper more eloquently than did we. For any target condition, we agree that strategy 4 should always be at least as good as strategy
3; however, strategy 3 might be more user-friendly. Our point in comparing the two was to determine if, when diagnosing airflow limitation, the superiority of strategy 4 was so large as to offset the computational simplicity of strategy 3 . We found it was not. Therefore, clinicians may use the less-rigorous method for diagnosing airflow limitation without fear of clinically important inaccuracy. Likewise, strategy 3 (and therefore strategy 4) should always be superior to strategy 2 for any target condition. However, strategies 3 and 4 can be used by a clinician only when these results have been published from well-designed and executed studies. What should the clinician use if he or she can find LR results only for individual clinical examination items? If investigators have not studied multiple items or have not used logistic regression, then strategies 3 and 4 cannot be used. Strategies 1 and 2 then become reasonable alternatives.

Our comparing strategies 2 and 3 was not to see if strategy 2 was better than 3 , but to see how much worse it was. Was strategy 2 so much worse than strategy 3 or 4 that it should never be considered an alternative? For the empiric example, we could not exclude potentially important differences with our sample size, but strategy 2 will yield a reasonable approximation when results needed for strategies 3 and 4 are not available.

Likewise, strategy 1 should always perform worse than strategies 3 and 4; however, it might be a reasonable alternative if the results of logistic regression analyses are not available to the clinician. Again, the question was not to see if strategy 1 was superior to logistic regression, but to see how much worse it was. For airflow limitation, we found that strategy 1 was substantially worse. Therefore, clinicians should be wary of using this approach to all clinical decision making and should avoid this approach altogether for airflow limitation.

We used logistic regression as an example of a technique for mathematically adjusting for nonindependence. We chose logistic regression because we thought it was presented more commonly in the literature and was more likely to be used by clinicianinvestigators. The best approach for variable reduction and correction for nonindependence was beyond the scope of our article. Additionally, we feared that if we used too many methods, people would accuse us of taking CART blanche.-Donald R. Holleman, JR., MD, Lexington VAMC and Department of Medicine, University of Kentucky, and DAVID L. Simel, MD, MHS, Center for Health Services Research in Primary Care, Durham VAMC, and Department of Medicine, Duke University Medical Center, NC.

\section{Quality in HIV/AIDS Care}

To the Editor:- I read with interest the article in the March 1997 issue by Markson et al. ${ }^{1}$ and the accompanying editorial by Holmes $^{2}$ regarding generalist-versus-specialist care of HIV. I agree with Holmes that in providing quality care for persons with HIV, current experience with HIV patients and an ability to keep up with the ever-changing status of HIV therapies are more important than past training per se. However, I would argue that what distinguishes an HIV "specialist" is this ongoing commitment of substantial time and effort, a commitment that may not be realistically possible for generalists who are already busy keeping up with general medicine and the rest of their patients. 
Maintaining a truly current knowledge base in the rapidly changing area of HIV therapy is difficult enough for any physician, even with new resources such as the Internet.

For example, I checked the Internet site http://www.critpath. org/soc10.htm. "a regularly updated standard of care," ${ }^{2}$ and found it was last updated October 23, 1996, making it 6 months old. Since that time two new drugs have been approved for HIV, and a major treatment trial, ACTG 320, was stopped early due to a significant difference in mortality between treatment arms. A standard of care for treatment of HIV even 6 months old is potentially already outdated. Moreover, the extensive literature cited by Holmes showing better care of HIV by specialists actually underestimates the current importance of up-to-date knowledge in managing HIV patients, as these studies were all conducted before the advent of protease inhibitors, highly active antiretroviral therapy, and viral load monitoring.

I agree with the qualities listed by Holmes as important components of a clinic caring for HIV patients, ${ }^{2}$ and would add to these a formal patient education program. Though patient education and medication compliance are important in all conditions, they are critical in treating HIV because of the high risk and consequences of viral drug resistance and cross-resistance. Development of a comprehensive care clinic involves the commitment of extensive multidisciplinary personnel and resources and in many communities has already been accomplished in the form of HIV specialty clinics. Such a system could be duplicated at each general medicine clinic that sees HIV patients. Or conversely, HIV patients could be referred to a specialty clinic that already has such a system complete with experienced and knowledgeable providers, without duplication of personnel and resources.

The question is not whether, with investment of enough time, effort, and money, high-quality comprehensive care of HIV infection could be provided by generalists. Rather, the question (with an answer that may depend on local factors) is whether this is any longer the most efficient way to provide such care.MichaEl Frank, MD, Indiana University School of Medicine, Indianapolis.

\section{REFERENCES}

1. Markson LE, Turner BJ, Cocroft J, Houchens R, Fanning TR. Clinic services for persons with AIDS: experience in a high prevalence state. J Gen Intern Med. 1997;12:141-9.

2. Holmes WC. Quality of HIV/AIDS care: specialty-related or experience-related? J Gen Intern Med. 1997;12:195-7.

In reply:-I agree with Dr. Frank's assertion that maintaining a current knowledge base about HIV/AIDS therapies is paramount if physicians are to provide quality HIV/AIDS care. Evolving practice patterns, however, are likely to drive more of the care of HIV seropositive individuals to generalists, perhaps even to generalists inexperienced in HIV/AIDS disease management. Given these increasingly likely developments, augmenting generalists' HIV/AIDS care experience and providing means by which generalists can maintain therapeutic knowledge are going to be two of many challenges we must face in ensuring quality care. The Internet is only one potential element, albeit integral, to providing solutions to the challenges at hand. Additional, equally integral elements are also required, and Dr. Frank, myself, and numerous others have discussed these.
As for the Internet, however, we must expand our view of how it might be used. To begin with, the use of numerous web sites is recommended. I suggested four sites. Regularly perusing more than just the standard-of-care site (for example, the "In the news" section of the http://www.aidsnyc.org/network site) would have alerted the generalist to the closure of ACTG 320, the warning regarding ACTG 290 (about unexplained drops in CD4 cell counts with AZT/d4T combination therapy), the approval of nelfinavir for use in adults and children, the approval of ritonavir for use in children, and the start of clinical trial recruitment for the new ganciclovir prodrug (RS-79070), to name only a few developments since October 1996. Perusing another of the sites, http:// www.projinf.org, would have alerted the generalist to recent case reports of a potential interaction between ritonavir and the recreational drug Ecstacy, or information about antivirograms.

The Internet may also be a useful tool in ways other than web-site information gathering. Consultation with specialists or specialist profiling of HIV/AIDS patient panels (with e-mail prompting sent to generalists providing substandard care), all via the Internet, are two of many other uses. Fortunately, the appropriate and ingenuous use of Internet resources may provide invaluable methods by which quality HIV/AIDS care can be achieved regardless of who provides that care.-William C. HoLmes, MD, Center for Clinical Epidemiology and Biostatistics, University of Pennsylvania, Philadelphia.

\section{Effect of Subspecialty Training and Experience on Agreement with HIV Guidelines}

To the Editor:-Recent studies have indicated that increased provider experience with care of HIV disease is associated with improved outcomes. Kitahata et al. demonstrated that physicians who had treated five or fewer patients with AIDS had less success than physicians who had treated more than five patients. ${ }^{1}$

Since 1994, the Center for Bio-medical Communication (CBC) has conducted annual surveys of physicians providing care to HIV-infected patients to assess current diagnostic, therapeutic, and preventive trends among providers. We analyzed provider responses to questions in the 1994-1996 CBC surveys that queried participants on practice patterns related to issues covered by one of the published federal guidelines ${ }^{2-5}$ and compared agreement with recommendations by provider experience (number of HIVinfected persons seen per year) and training (general internal medicine/family practice or infectious diseases).

A list of more than 12,000 physicians who had prescribed zidovudine at least once was obtained from a pharmacy supplier. Physicians were divided into quintiles, and a mailing was sent to a random sample of 1,000 physicians whose total number of zidovudine prescriptions was between $60 \%$ and $80 \%$ of the maximum (fourth of five quintiles). Respondents were invited to participate on a regular basis in surveys on various issues related to the care of HIV-infected persons, and were reimbursed for their time. Questionnaires were distributed to the respondent group three to six times per year; the same group of respondents was polled for each sampling.

Of the 1,000 physicians invited to participate by letter, 95 agreed to participate in the surveys. The median number of HIV- 
infected patients seen per year was 50 (range 3-2,000). Of the 95 respondents, $48(51 \%)$ were general internists or family practitioners (generalists) and 47 (49\%) were infectious diseases specialists (specialists). The median number of HIV-infected patients seen per year by the generalists was 20 (range $3-2,000$ ), while the median number of HIV-infected patients seen per year by the specialists was 100 (range 3-2,000) $(p<.01)$. Overall, $427(80 \%)$ of 536 responses were in accordance with Public Health Service "A" or "E" recommendations ("standard of care"). As seen in Table 1, there were no significant differences between the specialists and generalists on 8 of 10 recommendations; the specialists were significantly less likely than the generalists to recommend antifungal prophylaxis $(p<.02)$ or routine cervical screening $(p<.01)$. When providers were stratified by annual number of HIV-infected patients seen, those seeing more than 50 patients per year were less likely (88\%) than those seeing 3 to 50 patients (100\%) to recommend influenza vaccine.

Because the sample of respondents was self-selected, these persons may have been more highly motivated to provide HIV optimal care than among the general HIV provider population and more likely to be familiar with U.S. Public Health Service guidelines than nonresponders. The low response rate also limits the generalizability of the conclusions. Nonetheless, these results suggest that among physicians with a moderate amount of experience in the care of HIV-infected patients, there is a high level of awareness of U.S. Public Health Service recommendations, and there are a few differences when physicians are stratified by specialty training or experience. We suggest that previous reports of failure to provide adequate care to HIV-infected persons may result from poor conformity with guidelines by physicians with minimal experience in caring for HIV-infected persons. Physicians with moderate experience appear likely to provide care in good compliance with "standard of care" recommendations, regardless of subspecialty training.-C. RoBerT HoRSBURGH, JR., MD, Emory University School of Medicine, Atlanta, Ga.; Fred M. GoRdin, MD, Georgetown University, Washington, DC; SHARON SchaIER, and Roberta Silver, Center for Biomedical Communication, Dumont, $N J$.

\section{REFERENCES}

1. Kitahata MM, Koepsell TD, Deyo RA, Maxwell CL, Dodge WT, Wagner EH. Physicians' experience with the acquired immunodeficiency syndrome as a factor in patients' survival. $\mathrm{N}$ Engl $\mathrm{J}$ Med. 1996;334:701-6.

2. Sande MA, Carpenter CCJ, Cobbs CG, et al. Antiretroviral therapy in adult HIV-infected patients: recommendations from a state-ofthe-art conference. JAMA. 1993;270:2583-9.

3. Centers for Disease Control. Recommendations of the USPHS task force on the use of zidovudine to reduce perinatal transmission of HIV. MMWR. 1994;43(RR-11):1-20.

4. Centers for Disease Control. USPHS recommendations for HIV counseling and voluntary testing for pregnant women. MMWR. 1995;44(RR-7): 1-11.

5. Centers for Disease Control. USPHS/IDSA guidelines for the prevention of opportunistic infections in persons infected with human immunodeficiency virus: a summary. MMWR. 1995;44(RR-8):1-34.

Table 1. Response of Generalists and Infectious Disease Specialists to Questions About HIV Treatment

\begin{tabular}{ccccccc}
\hline \hline Question* & $\begin{array}{c}\text { Generalists } \\
\text { (yes/total) }\end{array}$ & $\begin{array}{c}\text { Specialists } \\
\text { (yes/total) }\end{array}$ & $p$ Value & $\begin{array}{c}\text { 1-50 Patients } \\
\text { per Year } \\
\text { (yes/total) }\end{array}$ & $\begin{array}{c}51+\text { Patients } \\
\text { per Year } \\
\text { (yes/total) }\end{array}$ & $p$ Value \\
\hline 1. & $6 / 47$ & $5 / 45$ & 0.81 & $5 / 48$ & $6 / 41$ & 0.55 \\
2. & $46 / 48$ & $43 / 46$ & 0.67 & $46 / 46$ & $37 / 42$ & 0.02 \\
3. & $46 / 48$ & $45 / 46$ & 1.00 & $48 / 49$ & $41 / 42$ & 1.00 \\
4. & $45 / 46$ & $46 / 46$ & 1.00 & $46 / 47$ & $42 / 42$ & 1.00 \\
5. & $31 / 45$ & $28 / 46$ & 0.43 & $28 / 46$ & $30 / 42$ & 0.30 \\
6. & $23 / 45$ & $15 / 42$ & 0.15 & $18 / 45$ & $18 / 40$ & 0.80 \\
7. & $35 / 41$ & $43 / 45$ & 0.14 & $43 / 47$ & $39 / 43$ & 1.00 \\
8. & $45 / 47$ & $43 / 45$ & 1.00 & $46 / 47$ & $41 / 43$ & 1.00 \\
9. & $44 / 44$ & $36 / 44$ & 0.01 & $40 / 44$ & $36 / 40$ & 1.00 \\
10. & $21 / 47$ & $6 / 45$ & 0.01 & $17 / 47$ & $9 / 43$ & 0.11 \\
\hline
\end{tabular}

*1. Based on your clinical experience and/or published data, do you feel that decreased exposure to environmental sources of Mycobacterium avium complex is a reasonable prophylactic measure? (U.S. Public Health Service [USPHS] grade E)

2. Do you routinely vaccinate your HIV-infected patients against influenza virus? (USPHS grade C)

3. Do you routinely vaccinate your HIV-infected patients against Pneumococcus? (USPHS grade B)

4. Do you believe it is appropriate to initiate AZT therapy in pregnant HIV-infected patients in order to reduce the rate of perinatal transmission? (USPHS grade A)

5. Do you advise pregnant females to be HIV tested? (USPHS grade A)

6. Do you employ alternative toxoplasmosis prophylaxis in your HIV-infected patients who are seropositive for Toxoplasma but intolerant of TMP/SMX? (USPHS grade A)

7. Do you routinely perform TB skin testing on all your asymptomatic HIV-infected patients? (USPHS grade A)

8. Based on your clinical experience and/or review of the literature, do you consider combination antiretroviral therapy of benefit to your HIVinfected patients? (USPHS grade, not rated)

9. Do you routinely perform cervical dysplasia screening during initial gynecological examinations of your HIV-infected patients? (USPHS grade A)

10. Do you attempt routine prophylaxis for deep fungal infections in your HIV-infected patients? (USPHS grade C) 